\title{
Multiple Gouty Tophi with Bone Erosion and Destruction: A Report of an Early-onset Case in an Obese Patient
}

\author{
Reiko D Hayashi ${ }^{1}$, Masaya Yamaoka ${ }^{1}$, Hitoshi Nishizawa ${ }^{1}$, Shiro Fukuda ${ }^{1}$, Yuya Fujishima ${ }^{1}$, \\ Takekazu Kimura ${ }^{1}$, Jyunji Kozawa ${ }^{1}$, Shunbun Kita ${ }^{2}$, Taka-aki Matsuoka ${ }^{1}$, Michio Otsuki ${ }^{1}$, \\ Akihisa Imagawa ${ }^{1}$, Kimiyoshi Ichida ${ }^{3}$, Atsuo Taniguchi ${ }^{4}$, Norikazu Maeda ${ }^{2}$, \\ Tohru Funahashi ${ }^{2}$ and Iichiro Shimomura ${ }^{1}$
}

\begin{abstract}
A 27 year-old severely obese man (BMI, 35.1) had hyperuricemia and multiple gouty tophi with bone erosion and destruction, resulting in gait disturbance for 6 years after the early onset of gout at 21 years of age. His hyperuricemia was associated with hyperinsulinemia in obesity and a genetic variant of the ABCG2 gene. In addition, multiple gouty tophi with bone erosion and destruction might have been caused by hypoadiponectinemia and the elevation of the patient's pro-inflammatory cytokine (IL-1 $\beta$ ) level with the accumulation of visceral fat. In this case, bone and Ga-67 scintigraphy were useful for detecting the location and magnitude of gouty tophi.
\end{abstract}

Key words: hyperuricemia, obesity, multiple gouty tophi, bone erosion and destruction, bone and Ga-67 scintigraphy

(Intern Med 56: 1071-1077, 2017)

(DOI: 10.2169/internalmedicine.56.7923)

\section{Introduction}

Long-term hyperuricemia leads to gouty arthritis. Hyperuricemia is triggered by several genetic factors as well as environmental factors such as the overconsumption of purinerich foods and alcohol. Recently, the prevalence of obesity and obesity-related disease, including hyperuricemia has increased (1).

On the other hand, gouty tophi formation is caused by elevated serum uric acid levels, long-term gouty arthritis and the non-treatment period, as reported by many studies $(2,3)$. The prevalence of hyperuricemia and gouty arthritis has increased in association with the degree of serum urate elevation in comparison to the previous decades. In contrast, since hyperuricemia can be effectively controlled by urate lowering therapy, opportunities to treat multiple gouty tophi are decreasing. We herein report the case of a severe obese patient who showed an early-onset gout attack and multiple gouty tophi with bone erosion and destruction that developed over a short period.

\section{Case Report}

A 27-year-old man complaining of bilateral knee pain and gait disturbance was admitted to our hospital. He had been obese since childhood. His growth was normal and psychological disorders, including self-mutilation behavior had not been observed. He had drunk alcohol by chance during the previous 7 years. A member of his family had hyperuricemia without gout. The detailed information of his family was unclear. At 21 years of age, he first displayed severe knee pain and gout was suspected by an orthopedist. At the time, he was treated with a painkiller but he had not received any further treatment. Since then, he sometimes suffered from pain. He had a history of excess dietary purine

\footnotetext{
${ }^{1}$ Department of Metabolic Medicine, Graduate School of Medicine, Osaka University, Japan, ${ }^{2}$ Department of Metabolism and Atherosclerosis, Graduate School of Medicine, Osaka University, Japan, ${ }^{3}$ Department of Pathophysiology, Tokyo University of Pharmacy and Life Sciences, Japan and ${ }^{4}$ Institute of Rheumatology, Tokyo Women's Medical University, Japan. Received for publication June 28, 2016; Accepted for publication August 17, 2016 Correspondence to Dr. Masaya Yamaoka, myamaoka@imed2.med.osaka-u.ac.jp
} 
a)

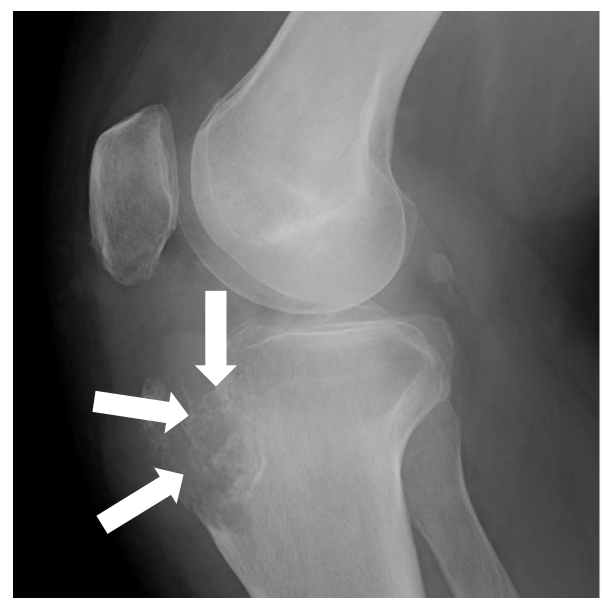

b)

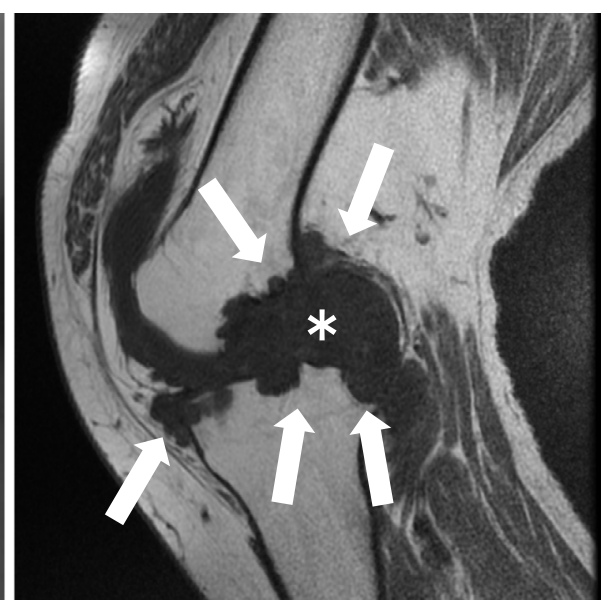

Figure 2. a) A lateral radiograph of the right knee joint. b) Sagittal T1-weighted magnetic resonance imaging of the right knee joint.

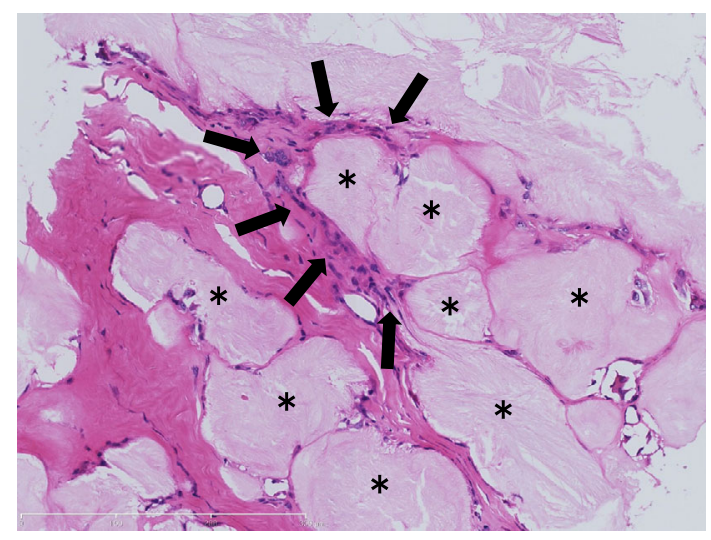

Figure 3. The histological examination of a punch biopsy specimen from the patient's right elbow. A representative Hematoxylin and Eosin staining section is shown.

A plain radiograph of the right knee showed a punchedout lesion characterized by gouty tophi (Fig. 2a, arrows). T1-weighted magnetic resonance imaging (MRI) of the left knee, revealed that the nodule had a low signal intensity (Fig. 2b, asterisk), indicating bone erosion and destruction (Fig. 2b, arrows).

A needle biopsy specimen taken from his right elbow showed deposits of an amorphous material that consisted of urate crystals (Fig. 3, asterisk) surrounded by granulomatous inflammation (Fig. 3, arrows). These histological features were compatible with a gouty tophus.

${ }^{99 \mathrm{~m}}$ Tc HMDP bone scintigraphy showed severe accumulation in the joints of both knees and ankles and in the right elbow joint and the left second metacarpophalangeal joint. ${ }^{99 \mathrm{~m}}$ Tc HMDP bone scintigraphy also revealed moderate accumulation in both sternoclavicular and shoulder joints and the right carpometacarpal joint (Fig. 4a). Ga-67 citrate scintigraphy showed the abnormal accumulation in similar sites, which corresponded to the abnormalities on ${ }^{99 \mathrm{~m}} \mathrm{Tc}$ HMDP bone scintigraphy (Fig. 4b). Abdominal echography revealed right renal calculi of approximately $1 \mathrm{~cm}$ in diameter.

The 24-hour urinary uric acid excretion (UUAE) of the patient ranged from 381 to $792 \mathrm{mg}$ /day during hospitalization. Thus, the UUAE values were not as high as the cut-off point $\left(880 \mathrm{mg} /\right.$ day/1.73 $\left.\mathrm{m}^{2}\right)$ proposed in a previous study (4). On the other hand, the renal clearance of uric acid (CUA) and the renal clearance of uric acid per creatinine clearance (fractional excretion of uric acid, FEUA) were reduced (CUA 1.91 to $4.15 \mathrm{~mL} / \mathrm{min}$, FEUA 1.72 to $2.28 \%$, respectively) (Fig. 5a). The plasma levels of hypoxanthine and xanthine were $0.028 \mathrm{mg} / \mathrm{dL}$ (reference value, $0-0.05 \mathrm{mg} / \mathrm{dL}$ ) and $0.0088 \mathrm{mg} / \mathrm{dL}$ (reference value, $0-0.31 \mathrm{mg} / \mathrm{dL}$ ), respectively. The HPRT gene and its associated activity were normal. Genetic variants of the ABCG2 genes (c.376C $>$ T, c.421C >A) and SLC16A9/MCT9 (c.773C >A) were detected, whereas other serum uric acid-related genes such as Uromodulin, SCL17A1NPT1, SLC17A3/NPT4, and ABCC4/ MRP4 gene mutations were not detected (5-8). These findings were likely to be associated with the elevation of the patient's serum level of uric acid.

After hospitalization, the patient first underwent diet therapy $(2,000 \mathrm{kcal} /$ day $)$ without medication for hyperuricemia. However, his serum UA levels remained high. Thus, on the 40th day of hospitalization, the use of pharmacological urate-lowering therapy was initiated. Because of his renal calculi, treatment with a xanthine oxidase inhibitor (febuxostat, $10 \mathrm{mg}$ per day) was initiated (Fig. 5a). After discharge, the dose was increased, and his UA level gradually decreased. However, his UA level remained at $8.5 \mathrm{mg} / \mathrm{dL}$ despite the use of febuxostat (60 mg per day). On day 286, additional treatment with benzbromarone (25 mg per day) was initiated because his FEUA had remained low. His FEUA increased to $5.25 \%$ and his UA level decreased to 6.1 $\mathrm{mg} / \mathrm{dL}$ after the use of benzbromarone (50 $\mathrm{mg}$ per day) (Fig. 5b and Table 2). 
a)

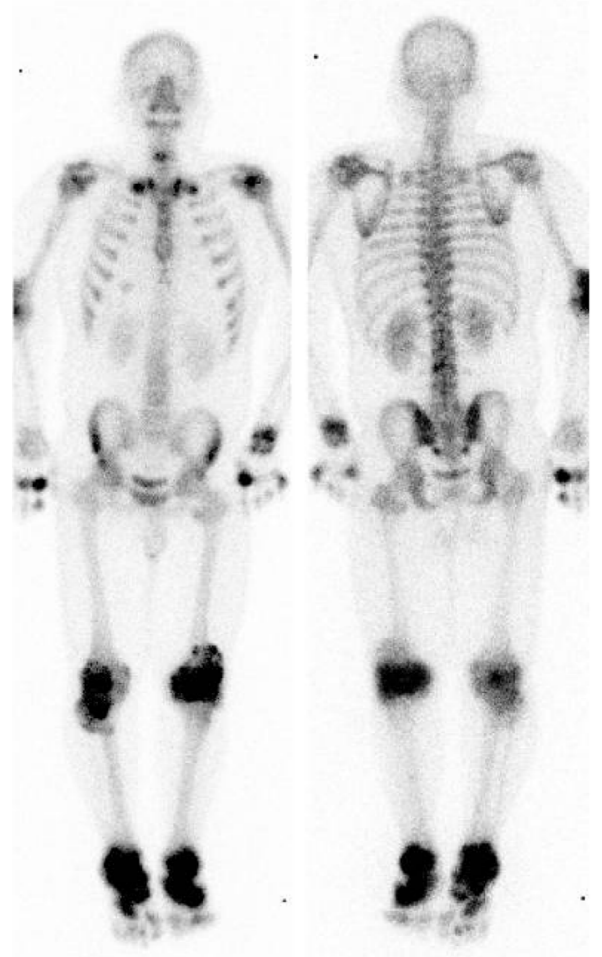

b)

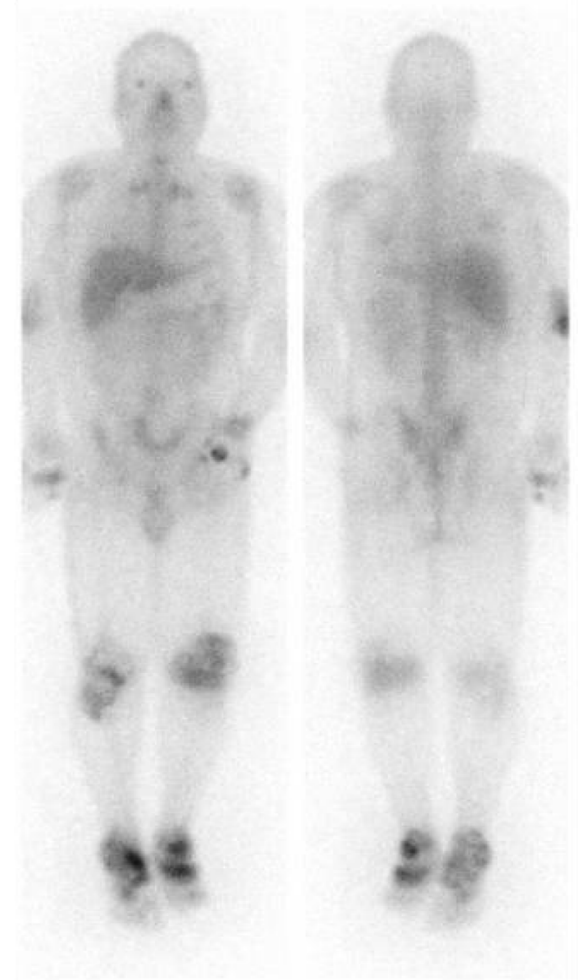

Figure 4. Whole body scintigraphy. a) ${ }^{99 \mathrm{~m}}$ Tc HMDP bone scintigraphy. b) Ga-67 citrate scintigraphy.

\section{Discussion}

The clinical course of this case provided two interesting findings. The first finding was the early onset and relatively acute progression of gout. The second was multiple gouty tophi with bone erosion and destruction. Patients with multiple gouty tophi are rarely encountered because hyperuricemia can now be effectively treated. However, this patient developed multiple gouty tophi over the course of only 6 years. Moreover, it was also interesting that inflammation and bone lesions due to gouty tophi could be detected throughout the whole body on nuclear images.

The etiology of the patient's severe hyperuricemia and gout was considered to include mutations of his uric acid transporters and severe obesity. In the present case, an ABCG2 gene mutation and a variant of SLC16A9/MCT9 were detected. Matsuo et al. reported that ABCG2 gene mutations were present in $78.6 \%$ of gout patients, and that the presence of the minor alleles of Q126X and Q141K leads to a remarkable decrease in the function of ABCG2. These dysfunctional genotype combinations lead to a more than $75 \%$ reduction in the function of $\mathrm{ABCG} 2$, and markedly increase the gout risk, conferring an OR of 25.8 (9). In addition, Matsuo et al. reported that severe ABCG2 dysfunction ( $25 \%$ of function) leads to a significant increase in the risk of early-onset gout, with an OR of 22.2 (10). The age of onset in patients with severe ABCG2 dysfunction is 6.5 years younger than that in patients with full function (10).
Furthermore, Nakayama et al. reported that a genetic variant of SLC16A9/MCT9 slightly increased the risk of hyperuricemia and gout (11), suggesting that the patient's SLC16A9/MCT9 mutation had a limited role in his hyperuricemia and gout. Although ABCG2 dysfunction has been reported to increase UUAE (12), the UUAE of the patient in the present case did not increase during the clinical course. Thus, it is suggested that other mechanisms, in addition to ABCG2 dysfunction, were important in the uric acid metabolism of the present case. We therefore hypothesized that that the patient's hyperuricemia and gout might have also been caused by his severe obesity. Hyperuricemia has previously been reported to be associated with the impaired renal clearance of uric acid in severely obese subjects $(13,14)$. In addition, we reported that both the visceral fat area and the serum adiponectin concentration were significant explanatory variables for serum uric acid levels (15). Moreover, the change in the visceral fat area was corresponded to the changes in serum uric acid levels over a one-year period in general subjects (15). Several studies have reported that hyperinsulinemia might contribute to a mechanism of impaired renal clearance of uric acid in obese subjects $(14,16,17)$. In the present patient, it is suggested that severe obesity with visceral fat accumulation and hyperinsulinemia were considerably associated with the etiology of hyperuricemia through the decreased renal clearance of uric acid (CUA and EFUA). Since, ABCG2 dysfunction is reported to decrease the renal clearance of uric acid (18), the patient's hyperuricemia might have been partly affected by the decreased renal clear- 
a)

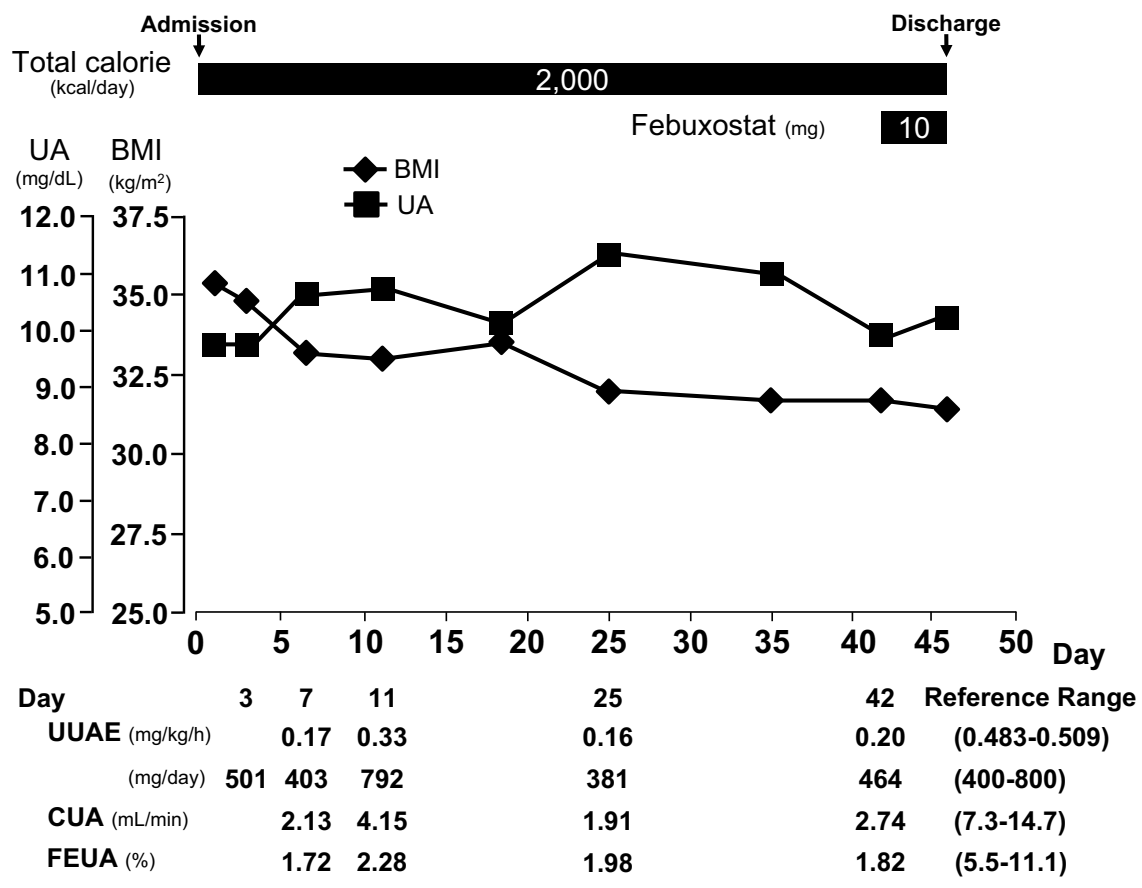

b)
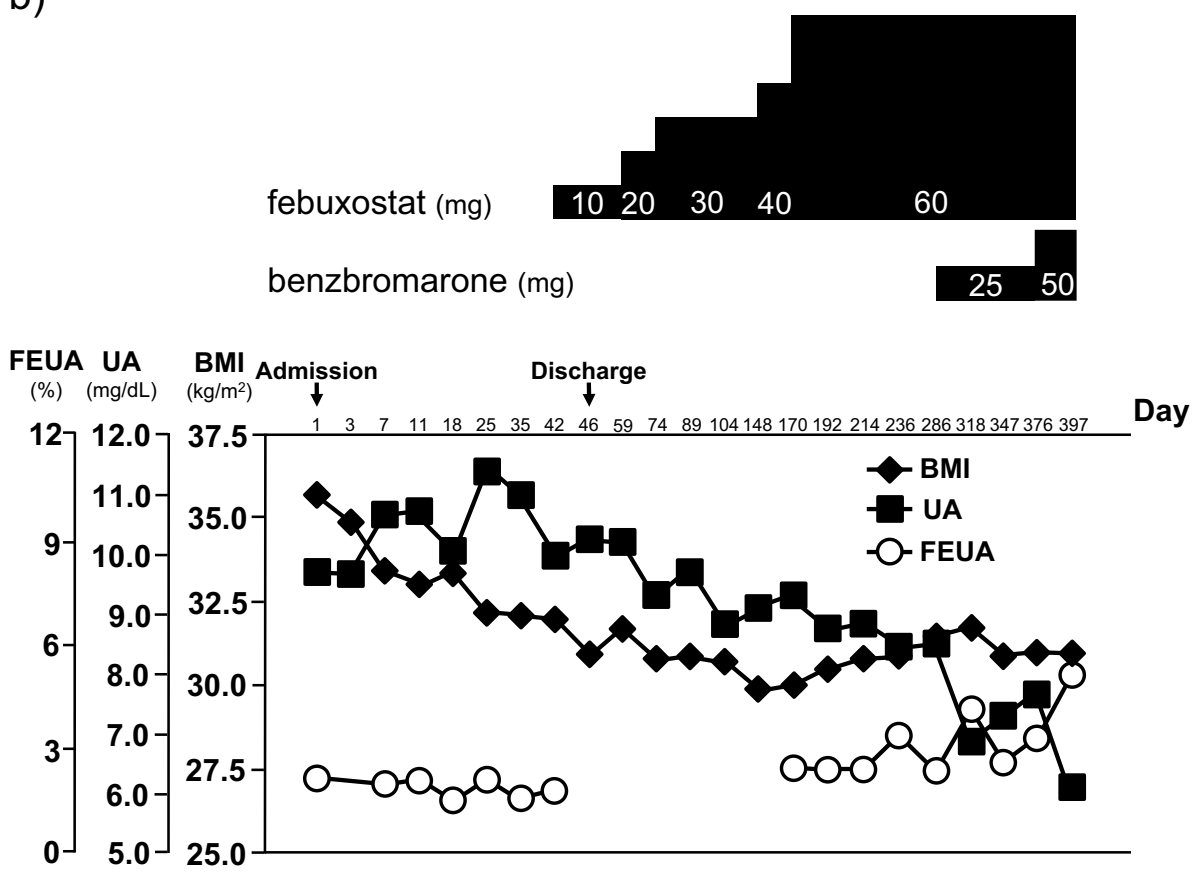

Figure 5. A summary of the clinical course. a) The clinical course in the hospital. b) The clinical course in the overall treatment period.

Table 2. Clinical Course of Indices Related to Excretion of Uric Acid.

\begin{tabular}{lccccccc}
\hline Day & 7 & 11 & 25 & 42 & 376 & 397 & \\
\hline & & & & & & & Reference Range \\
UUAE $(\mathrm{mg} / \mathrm{kg} / \mathrm{h})$ & $\mathbf{0 . 1 7}$ & $\mathbf{0 . 3 3}$ & $\mathbf{0 . 1 6}$ & $\mathbf{0 . 2 0}$ & $\mathbf{0 . 1 9}$ & $\mathbf{0 . 3 4}$ & $\mathbf{( 0 . 4 8 3 - 0 . 5 0 9 )}$ \\
CUA $(\mathrm{mL} / \mathrm{min})$ & $\mathbf{2 . 1 3}$ & 4.15 & 1.91 & $\mathbf{2 . 7 4}$ & $\mathbf{3 . 6 1}$ & $\mathbf{2 . 4 0}$ & $\mathbf{( 7 . 3 - 1 4 . 7 )}$ \\
FEUA $(\%)$ & $\mathbf{1 . 7 2}$ & $\mathbf{2 . 2 8}$ & $\mathbf{1 . 9 8}$ & $\mathbf{1 . 8 2}$ & $\mathbf{2 . 5 7}$ & $\mathbf{5 . 2 5}$ & $\mathbf{( 5 . 5 - 1 1 . 1 )}$ \\
\hline
\end{tabular}

ance derived from his $\mathrm{ABCG} 2$ dysfunction, in addition to his severe obesity.
In the present case, it was clinically interesting that multiple hot spots in whole body were detected on nuclear imag- 
ing, which showed the existence of inflammation (Ga-67 scintigraphy) and bone lesions ( ${ }^{99 \mathrm{~m}} \mathrm{Tc}$ HMDP bone scintigraphy) due to gout. Chowalloor et al. reported that bone scans are highly sensitive but not highly specific in detecting osseous abnormalities (19); although a few reports have already described the evaluation of gouty tophi on nuclear imaging (20-23). The scintigraphic findings in the present case were helpful for the assessment of the location and magnitude of gouty arthritis and tophi (19). Follow-up Ga-67 scintigraphy performed one year after discharge still showed multiple hot spots in the bilateral knee and ankle joints in spite the fact that there was no symptomatic gout attack. It is therefore possible that nuclear imaging is useful for assessing the recovery phase of gout after urate lowering therapy.

The mechanism through which gouty tophi with bone destruction developed in the present patient is considered to involve the activation of osteoclasts because of the elevation of bone resorption markers such as TRACP-5b. Dalbeth et al. demonstrated, through the use of cell culture experiments, that MSU crystals promoted the formation of osteoclasts through the inhibition of osteoprotegerin, which is known to suppress the differentiation of osteoclasts (24). In the present case, the elevation of the patient's bone resorption marker levels might imply that enhanced osteoclastogenesis was involved in the development of bone erosions and destruction. In addition, it is reported that increased levels of pro-inflammatory cytokines such as interleukin (IL)$1 \beta$ are also associated with the development of bone erosion and destruction (25-27). The levels of these cytokines are elevated in obese subjects. Thus, we suggested that severe obesity-related pro-inflammatory cytokine (IL-1 $\beta$ ) elevation partially contributed to development of bone erosion and destruction in the present case. Furthermore, adiponectin is also associated with the bone metabolism. Oshima et al. reported that adiponectin increased bone mass by both suppressing osteoclastogenesis and activating osteoblastogenesis (28). In the present case, the serum adiponectin level was decreased; thus, hypoadiponectinemia might have also been partially involved in bone absorption.

Collectively, in addition to the patient's ABCG2 (and SCL16A9/MCT9) gene mutations, his severe obesity and visceral fat accumulation, hyperinsulinemia and hypoadiponectinemia might have led to the development of severe hyperuricemia and the early onset of multiple gouty tophi with bone lesions, which resulted in gait disturbance, over a period of only 6 years. Thus, the early diagnosis of gout and the early initiation of an appropriate therapy for hyperuricemia are very important when orthopedists and physicians encounter patients with severe obesity and arthritis.

\section{Conclusion}

We reported an unusual early-onset case (21 year of age) of multiple gouty tophi including bone erosion and destruction a short period after ( 6 years) the initial manifestation of gouty arthritis. It is possible that the combination of severe obesity, hypoadiponectinemia and the mutations of genes involved in uric acid transport led to severe hyperuricemia and multiple gouty tophi with bone lesions in the present case. In the present case involving a patient with gouty arthritis and multiple gouty tophy, whole body scintigraphy was useful for investigating the patient's condition and for assessing the location and magnitude of his multiple gouty tophi.

The authors state that they have no Conflict of Interest (COI).

\section{Acknowledgement}

We thank Ikuo Mineo and Yu Tsushima for the helpful discussion on this report.

\section{References}

1. Matsuzawa Y. Adiponectin: a key player in obesity related disorders. Curr Pharm Des 16: 1896-1901, 2010.

2. Gutman AB. The past four decades of progress in the knowledge of gout, with an assessment of the present status. Arthritis Rheum 16: 431-445, 1973

3. Nakayama DA, Barthelemy C, Carrera G, Lightfoot RW Jr, Wortmann RL. Tophaceous gout: a clinical and radiographic assessment. Arthritis Rheum 27: 468-471, 1984.

4. Simkin PA. New standards for uric acid excretion and evidence for an inducible transporter. Arthritis Rheum 49: 735-736, 2003.

5. Gibson T. Hyperuricemia, gout and the kidney. Curr Opin Rheumatol 24: 127-131, 2012.

6. Jutabha P, Anzai N, Kitamura K, et al. Human sodium phosphate transporter 4 (hNPT4/SLC17A3) as a common renal secretory pathway for drugs and urate. J Biol Chem 285: 35123-35132, 2010.

7. El-Sheikh AA, van den Heuvel JJ, Koenderink JB, Russel FG. Effect of hypouricaemic and hyperuricaemic drugs on the renal urate efflux transporter, multidrug resistance protein 4. Br J Pharmacol 155: 1066-1075, 2008.

8. Torres RJ, de Miguel E, Bailén R, Banegas JR, Puig JG. Tubular urate transporter gene polymorphisms differentiate patients with gout who have normal and decreased urinary uric acid excretion. J Rheumatol 41: 1863-1870, 2014.

9. Matsuo H, Takada T, Ichida K, et al. Common defects of ABCG2, a high-capacity urate exporter, cause gout: a function-based genetic analysis in a Japanese population. Sci Transl Med 1: 5ra11, 2009.

10. Matsuo $H$, Ichida $K$, Takada $T$, et al. Common dysfunctional variants in ABCG2 are a major cause of early-onset gout. Sci Rep 3: 2014, 2013.

11. Nakayama A, Matsuo H, Shimizu T, et al. Common missense variant of monocarboxylate transporter 9 (MCT9/SLC16A9) gene is associated with renal overload gout, but not with all gout susceptibility. Hum Cell 26: 133-136, 2013.

12. Ichida $K$, Matsuo $H$, Takada $T$, et al. Decreased extra-renal urate excretion is a common cause of hyperuricemia. Nat Commun $\mathbf{3}$ : 764, 2012.

13. Yamashita S, Matsuzawa Y, Tokunaga K, Fujioka S, Tarui S. Studies on the impaired metabolism of uric acid in obese subjects: marked reduction of renal urate excretion and its improvement by a low-calorie diet. Int J Obes 10: 255-264, 1986.

14. Facchini F, Chen YD, Hollenbeck CB, Reaven GM. Relationship between resistance to insulin-mediated glucose uptake, urinary uric acid clearance, and plasma uric acid concentration. JAMA 266: 3008-3011, 1991.

15. Tamba S, Nishizawa H, Funahashi T, et al. Relationship between 
the serum uric acid level, visceral fat accumulation and serum adiponectin concentration in Japanese men. Intern Med 47: 11751180,2008

16. Ter Maaten JC, Voorburg A, Heine RJ, Ter Wee PM, Donker AJ, Gans RO. Renal handling of urate and sodium during acute physiological hyperinsulinaemia in healthy subjects. Clin Sci (Lond) 92: 51-58, 1997.

17. Lee J, Lee JY, Lee JH, et al. Visceral fat obesity is highly associated with primary gout in a metabolically obese but normal weighted population: a case control study. Arthritis Res Ther 17: 79, 2015.

18. Matsuo H, Nakayama A, Sakiyama M, et al. ABCG2 dysfunction causes hyperuricemia due to both renal urate underexcretion and renal urate overload. Sci Rep 4: 3755, 2014.

19. Chowalloor PV, Siew TK, Keen HI. Imaging in gout: a review of the recent developments. Ther Adv Musculoskelet Dis 6: 131-143, 2014.

20. Fernandes A, Faria MT, Oliveira A, Vieira T, Pereira J. Bone scintigraphy in tophaceous gout. Eur J Nucl Med Mol Imaging 43: 1387-1388, 2016.

21. Goshen E, Schwartz A, Zwas ST. Chronic tophaceous gout: scintigraphic findings on bone scan. Clin Nucl Med 25: 146-147, 2000.

22. Coombs RJ, Pinsky ST, Padanilam TG. Bone scan findings of combined gout and septic arthritis in the same digit. Clin Nucl Med 26: 442-443, 2001.

23. Appelboom T, Emery P, Tant L, Dumarey N, Schoutens A. Evaluation of technetium-99m-ciprofloxacin (Infecton) for detecting sites of inflammation in arthritis. Rheumatology (Oxford) 42: 1179-1182, 2003.

24. Dalbeth N, Smith T, Nicolson B, et al. Enhanced osteoclastogenesis in patients with tophaceous gout: urate crystals promote osteoclast development through interactions with stromal cells. Arthritis Rheum 58: 1854-1865, 2008.

25. Schlesinger N, Thiele RG. The pathogenesis of bone erosions in gouty arthritis. Ann Rheum Dis 69: 1907-1912, 2010.

26. Mundy GR. Osteoporosis and inflammation. Nutr Rev 65: S147S151, 2007.

27. Chhana A, Dalbeth N. The gouty tophus: a review. Curr Rheumatol Rep 17: 19, 2015.

28. Oshima K, Nampei A, Matsuda M, et al. Adiponectin increases bone mass by suppressing osteoclast and activating osteoblast. Biochem Biophys Res Commun 331: 520-526, 2005.

The Internal Medicine is an Open Access article distributed under the Creative Commons Attribution-NonCommercial-NoDerivatives 4.0 International License. To view the details of this license, please visit (https://creativecommons.org/licenses/ by-nc-nd/4.0/).

(C) 2017 The Japanese Society of Internal Medicine http://www.naika.or.jp/imonline/index.html 